\title{
Fatigue performance of endodontically treated premolars restored with direct and indirect cuspal coverage restorations utilizing fiber-reinforced cores
}

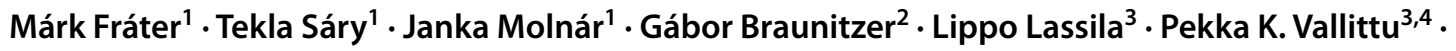 \\ Sufyan Garoushi ${ }^{3}$ (i)
}

Received: 5 July 2021 / Accepted: 23 November 2021 / Published online: 30 November 2021

(c) The Author(s) 2021

\begin{abstract}
Objectives The aim of this in vitro study was to investigate the fatigue survival and fracture behavior of endodontically treated (ET) premolars restored with different types of post-core and cuspal coverage restorations.

Materials and methods MOD cavities were prepared on 108 extracted maxillary premolars. During the endodontic treatment, all teeth were instrumented with rotary files (ProTaper Universal) to the same apical enlargement (F2) and were obturated with a matched single cone obturation. After the endodontic procedure, the cavities were restored with different post-core and overlay restorations ( $n=12$ /group). Three groups (A1-A3) were restored with either conventional composite core (PFC; control) or flowable short-fiber-reinforced composite (SFRC) core with/without custom-made fiber posts and without overlays. Six groups had similar post-core foundations as described above but with either direct PFC (B1-B3) or indirect CAD/CAM (C1-C3) overlays. Fatigue survival was tested for all restorations using a cyclic loading machine until fracture occurred or 50,000 cycles were completed. Kaplan-Meyer survival analysis was conducted, followed by pairwise post hoc comparisons. Results None of the restored teeth survived all 50,000. Application of flowable SFRC as luting-core material with fiber post and CAD/CAD overlays (Group C3) showed superior performance regarding fatigue survival $(p<0.05)$ to all the other groups. Flowable SFRC with fiber post and direct overlay (Group B3) showed superior survival compared to all other direct techniques $(p<0.05)$, except for the same post-core foundation but without cuspal coverage (Group A3).

Conclusions Custom-made fiber post and SFRC as post luting core material with or without cuspal coverage performed well in terms of fatigue resistance and survival when used for the restoration of ET premolars.

Clinical relevance The fatigue survival of direct and indirect cuspal coverage restorations in ET MOD premolars is highly dependent on whether the core build-up is fiber-reinforced or not. The combination of short and long fibers in the form of individualized post-cores seems to offer a favorable solution in this situation.
\end{abstract}

Keywords Premolars · Endodontically treated teeth $\cdot$ Short-fiber-reinforced composite $\cdot$ Fatigue survival $\cdot$ Indirect restoration $\cdot$ Fiber-reinforced post $\cdot$ Overlay

Márk Fráter and Tekla Sáry contributed equally to this work

Sufyan Garoushi

sufgar@utu.fi

1 Department of Operative and Esthetic Dentistry, Faculty of Dentistry, University of Szeged, Szeged, Hungary

2 dicomLAB Dental Ltd., Szeged, Hungary

3 Department of Biomaterials Science and Turku Clinical Biomaterials Center-TCBC, Institute of Dentistry, University of Turku, Turku, Finland

4 City of Turku Welfare Division, Oral Health Care, Turku, Finland

\section{Introduction}

Root canal treated (RCT) teeth are at an increased risk of failure-related fracture [1, 2]; therefore, the restorative material for their permanent restoration must be carefully chosen [3]. Dietschi et al. showed that the main reason for this increased risk is caries-related coronal destruction and the dentine loss that occurs during endodontic treatment [4]. These are quite significant in mesio-occluso-distal (MOD) cavities [5]. While a class I occlusal cavity causes only 5-20\% loss of relative cuspal stiffness [6, 7], an MOD cavity causes an average of $63 \%$ loss of relative cuspal stiffness 
[8]. This is related mainly to the loss of both marginal ridges $[9,10]$. As a result, fracture strength may be reduced by as much as $54 \%[11,12]$. Thus, intracoronal reinforcement in endodontically treated premolars with MOD cavities is essential to protect them against fracture [13, 14].

In the last 30 years, mainly fiber-reinforced composite (FRC) posts have been used to restore RCT premolars [15]. However, their actual reinforcing efficiency is still a matter of debate. Some studies managed to show increased fracture resistance when using FRC posts in endodontically treated premolar teeth [16-18], while others failed to do so [19, 20]. Yet other studies managed to show a positive effect only on failure patterns, but not on fracture resistance [21, 22].

The failure of conventional FRC posts to reinforce premolar teeth may have multiple reasons: their inadequate fit in the cervical region of the canal (leading to an increased amount of luting cement) [23, 24], poor bonding of the post material to the luting cement $[25,26]$, or even the biomechanically inadequate position of the fibers inside the canal [27]. Aiming to solve the above-mentioned shortcomings, the authors have proposed the Bioblock technique for the individualized restoration of irregular root canals [28-30]. In many cases, the Bioblock technique, utilizing either packable or flowable short fiber-reinforced composite (SFRC), has shown superior results compared to FRC posts [28, 29]. Furthermore, according to the latest research, those results may be further enhanced by using flowable SFRC as a luting material next to the FRC post inside the canal [31].

So far, cuspal coverage in the case of MOD cavities in root canal-treated teeth has been considered a mandatory safety measure by many [32-34]. However, with the development of individualized fiber-reinforced post and cores, the question arises whether cuspal coverage is necessary or not. It is also a yet unanswered question if there is a difference between direct (performed with particulate filled composite, PFC) and indirect cuspal coverage in this respect.

Thus, the purpose of this in vitro investigation was to evaluate whether a combination of an FRC post and flowable SFRC or flowable SFRC alone is ideal for the reinforcement of MOD endodontically treated premolars. In addition, the question of the effect of direct/indirect cuspal coverage on fatigue behavior was also investigated.

The null hypotheses were that (1) the tested individualized FRC post and core build-ups with and without cuspal coverage restorations would not differ from the control group in fatigue survival or (2) in their fracture pattern.

\section{Materials and methods}

The study conformed to the tenets of the Declaration of Helsinki, and it was approved by the Regional Human Biomedical Research Ethics Committee at the University of Szeged,
Hungary. All restorative materials used in this study are made by the same manufacturer and used according to the manufacturer's instructions.

One hundred and eight maxillary premolar teeth, extracted for periodontal or orthodontic reasons, were selected for this investigation. The newly extracted premolars were directly inserted in sodium hypochlorite (5.25\%) for $5 \mathrm{~min}$ and then kept in saline $(0.9 \%)$ for a maximum of 12 weeks at room temperature before use. After extraction, with the aid of hand scalers, the root surface was cleaned from the covered soft tissue. The teeth selection criteria regarding their integrity and radicular dimensions were the same as in our previous method [31]. Regarding coronal dimensions, $90 \%$ of the teeth ranged between 9 and $10 \mathrm{~mm}$ bucco-palatally, measured at the widest bucco-palatal dimension. The average mesio-distal dimension was between 7 and 7.5 for $90 \%$ of the samples. Ten percent maximum deviation was allowed in the remaining $10 \%$ of the samples. The height of the crowns was between 7.5 and $8 \mathrm{~mm}$ in $90 \%$ of the samples, and $+/ 0.5 \mathrm{~mm}$ deviation was allowed in the rest of the samples.

The teeth were randomly distributed across 9 study groups of 12 specimens each $(A 1=$ control, A2-3, B1-3, C1-3). Specimens with average dimensions (within the above-mentioned 90\%) were randomly distributed across the 9 groups, and specimens with non-average dimensions (the remaining 10\%) were evenly distributed across the same 9 groups. MOD cavity preparation and root canal treatment were performed by the same trained operator. A standardized MOD cavity was prepared using a round-end parallel diamond (881.31.014 FG, Brasseler USA Dental, Savannah, GA) with water cooling. So the cavity was prepared so that the buccopalatal width of the occlusal isthmus was one-third of the intercuspal width, and the proximal box width was half of the buccopalatal width of the crown. The gingival floor was located $1 \mathrm{~mm}$ above the cemento-enamel junction (CEJ). All internal angles were rounded and the cavosurface margins were at $90^{\circ}$. After finalizing the MOD cavity preparation, access cavity preparation was carried out with a round-end diamond bur (850-014 M SSWhite, Lakewood, NJ, USA) with water cooling, and root canal treatment was performed in the prepared teeth.

The working length was defined utilizing the direct method, that is, by subtracting $1 \mathrm{~mm}$ from the real root length, which was determined by introducing a number 10 K-file (Maillefer-Dentsply, Ballaigues, Switzerland) until it was visible through the apical foramen. The root canals were prepared using rotary ProTaper Universal files (Maillefer-Dentsply). The ProTaper sequence (S1, S2, F1, F2) was used for the preparation of the working length. Irrigation was done after every instrument with $2 \mathrm{ml}$ of $2.5 \%$ $\mathrm{NaOCl}$ solution, and the canal space was filled with irrigant during the instrumentation phase. After root canal cleaning 
and shaping, the roots were dried using $96 \%$ alcohol and paper points. Root canal obturation was done by matched single-cone obturation with a master cone (F2 gutta-percha, Maillefer-Dentsply) and sealer (AH plus; Dentsply De Trey $\mathrm{GmbH}$, Konstanz, Germany). The gutta-percha was cut back to the level of the orifice, and the access cavity was temporarily filled with Fuji Triage Pink (GC Europe, Leuven, Belgium). Fuji Triage Pink was applied to the apical part of the root to prevent leakage through the apex. The teeth were stored wet in an incubator (mco-18aic, Sanyo, Japan) for 1 week (at $37{ }^{\circ} \mathrm{C}, 100 \%$ relative humidity). After this, the temporary material was removed, and the MOD cavity including the access cavity was refreshened with a diamond bur.

In Groups A2-3, B2-3, and C2-3, post space preparation was carried out by a 1.2 GC Fiber Post drill to a depth of 6-mm apical from the root canal orifice, as proposed in one of our previous studies [31]. After cutting back the guttapercha, the root canal was washed with chlorhexidine and dried with paper points.

Finally, in all groups marked "B" and "C," all cusps were reduced by $2 \mathrm{~mm}$ of their original height.

All specimens received the same adhesive treatment. A Tofflemire (1101C 0.035, KerrHawe, Bioggio, Switzerland) matrix band was applied before the adhesive treatment of the cavity and the root canal, and the enamel was selectively acid-etched with $37 \%$ phosphoric acid for $15 \mathrm{~s}$ and washed with water. The coronal cavity and the root canal were rinsed with $2 \mathrm{ml}$ of water and dried with paper points and air. A dual-cure one-step self-etch adhesive system (G-Premio Bond and DCA, GC Europe) was used for bonding, according to the manufacturer's instructions, using a microbrush-X disposable applicator (Pentron Clinical Technologies, LLC, USA). Excess adhesive was eliminated by suction drying (Evacuation Tip-Starryshine, Anaheim, CA, USA) applied approximately $0.5 \mathrm{~cm}$ from the occlusal cavity (without contact). Excess adhesive resin at the bottom of the canal was eliminated using a paper point. The adhesive was lightcured for $60 \mathrm{~s}$ using an Optilux 501 quartz-tungsten-halogen light-curing unit (Kerr Corp., Orange, CA, USA). The lightcuring tip was always in close contact (1-2 $\mathrm{mm}$ ) with the tooth surface. The average power density of the light source, measured with a digital radiometer (Jetlite light tester, J. Morita USA Inc. Irvine, CA, USA) before the bonding procedure, was $840 \pm 26.8 \mathrm{~mW} / \mathrm{cm}^{2}$.

In the control group and group B1, the missing interproximal walls were built up with conventional composite (G-aenial Universal Injectable, shade A3, GC Europe), while in groups A2, A3, B2, and B3, the missing interproximal walls were built up with flowable SFRC (everX Flow, dentine shade, GC Europe) using the centripetal technique, thus transforming the MOD cavity into a class I cavity. This interproximal wall was light-cured for $40 \mathrm{~s}$.
The teeth/groups were restored according to different restorative approaches (see Fig. 1 and Table 1).

Group A1 = control group The cavities were restored with conventional PFC composite material (G-aenial Universal Injectable) applied with an oblique incremental technique. The material was placed in consecutive 2-mm-thick increments. Each increment was light-cured from the occlusal surface for $40 \mathrm{~s}$.

Group A2 The cavities and the 6-mm-deep post space were reconstructed with the Bioblock technique described by Fráter et al. [28], building a direct layered post and core from flowable SFRC. The thickness of the increments was approximately $4 \mathrm{~mm}$, and a microbrush-X disposable applicator (Pentron Clinical Technologies, LLC, USA) was used. A light-transmitting FRC post (1.2 mm GC Fiber post, GC Europe) was inserted into the post space to aid the transmission of the light to the apically positioned layers. The "light transmitting" post was withdrawn $0.5-1 \mathrm{~mm}$ from the surface of the uncured SFRC layer not to have direct contact with it. This apical layer was light-cured through the fiber post for $80 \mathrm{~s}$. The rest of the cavity was restored with two 4-mm-thick layers of flowable SFRC (everX Flow, bulk shade, GC Europe). The material was placed to a level according to the anatomy of the dentine, leaving $2 \mathrm{~mm}$ occlusally for the final PFC composite. These SFRC increments were light-cured from the occlusal surface for $40 \mathrm{~s}$. The last occlusal layer was conventional composite material (G-aenial Universal Injectable) covering the SFRC as described in Group A1.

Group A3 The teeth received a custom-made unidirectional FRC post (everSTICK POST, GC Europe). Before the adhesive treatment, the posts of $1.2-\mathrm{mm}$ diameter were tried in and cut to a length of $2 \mathrm{~mm}$ below the level of the occlusal cavity margins with sterile scissors. Luting of the posts and the core build-up was performed with flowable SFRC as described by Fráter et al. [31]. Flowable SFRC was applied in approx. 4-mm-thick layers into the post space. After insertion of the post, light-curing was performed for $60 \mathrm{~s}$. The coronal portion of the cavity was restored as described in Group A2.

Group B1 The cavities were restored with conventional PFC composite material (G-aenial Universal Injectable) as described in Group A1 to the level of the occlusal reduction. The previously reduced cusps were built back by conventional PFC composite with the aid of a silicon index.

Group B2 The cavities and the 6-mm-deep post space were reconstructed with flowable SFRC as in Group A2 to the level of reduction. The previously reduced cusps were built 
Fig. 1 Diagram showing the test groups (A1-C3) restored with different approaches with various post-core and overlay restorations. A1 (control), only PFC without overlay; A2, SFRC + PFC; $\mathrm{A} 3$, post $+\mathrm{SFRC}+\mathrm{PFC} ; \mathrm{B} 1$, $\mathrm{PFC}+$ direct overlay; $\mathrm{B} 2$,

SFRC + direct overlay; B3, post + SFRC + direct overlay; $\mathrm{C} 1, \mathrm{PFC}+$ indirect overlay; $\mathrm{C}$, $\mathrm{SFRC}+$ indirect overlay; $\mathrm{C} 3$, post + SFRC + indirect overlay




Table 1 Different post-core and cuspal coverage restorations $(n=12 /$ group)

\begin{tabular}{lll}
\hline Group & Post-core & Cuspal coverage \\
\hline A1 (control) & PFC core & No \\
A2 & SFRC & No \\
A3 & Post + SFRC & No \\
B1 & PFC core & Direct PFC \\
B2 & SFRC & Direct PFC \\
B3 & Post + SFRC & Direct PFC \\
C1 & PFC core & CAD/CAM \\
C2 & SFRC & CAD/CAM \\
C3 & Post + SFRC & CAD/CAM \\
\hline
\end{tabular}

back by conventional PFC composite with the aid of a silicon index.

Group B3 The cavities and the 6-mm-deep post space were reconstructed with a custom-made FRC post (everSTICK POST) as in Group A3 to the level of reduction. The previously reduced cusps were built back with the conventional composite material with the aid of a silicon index.

Group C1 The cavities were restored with conventional composite material as described in Group A1 (G-aenial Universal Injectable) in the form of a core build-up, leaving 2-mm space for the overlay on the occlusal and also on the interproximal surfaces.

Group C2 The cavities and the 6-mm-deep post space were reconstructed with flowable SFRC as in Group A2 to the level of reduction in the form of a core build-up, leaving 2-mm space for the overlay on the occlusal and also on the interproximal surfaces.

Group C3 The cavities and the 6-mm-deep post space were reconstructed with a custom-made FRC post (everSTICK POST) as in Group A3 in the form of a core build-up, leaving 2-mm space for the overlay on the occlusal and also on the interproximal surfaces.

Groups $\mathrm{C} 1, \mathrm{C} 2$, and $\mathrm{C} 3$ then received indirect CAD/CAM overlays according to the following steps:

After refining the cavity margins, a polyether impression (Permadyne, 3 M ESPE) was taken of each prepared specimen, using a simultaneous mixing technique according to the manufacturer's instructions. The impressions were poured with type IV dental stone (FUJIROCK, GC Europe) and 2-mm-thick CAD/CAM composite resin overlays (CERASMART 270, GC Europe) were prepared by the same technician for each prepared specimen.
Luting was performed as follows: the intaglio surface of the composite overlays was treated with hydrofluoric acid for $20 \mathrm{~s}$. After rinsing and drying, the overlays were silanized (G-Multi PRIMER, GC Europe) and dried. As for the teeth, the enamel margins were etched with $37 \%$ phosphoric acid for $30 \mathrm{~s}$, rinsed with water, and air-dried. The surface of the composite core was roughened with a diamond bur, and a bonding agent was applied on the core (G-Premio Bond). Then the same bonding agent was applied on the intaglio of the restoration and left undisturbed for $10 \mathrm{~s}$. Using an air syringe, the surface of the restoration was dried for $5 \mathrm{~s}$ with maximum air pressure. The overlays were luted with dual-cure resin cement (G-CEM LinkForce, GC Europe). The luting agent was applied onto the intaglio surface of the overlays, and the overlays were applied on the teeth under finger pressure until complete adaptation. After removing the excess material, glycerine gel (DeOx Gel, Ultradent Products Inc., Orange, CA, USA) was applied, and photopolymerization from each side for $40 \mathrm{~s}$ with Optilux 501 was performed.

Embedding and the simulation of the periodontal ligaments were carried out as described in our previous studies $[31,35]$. During the mechanical testing, the specimens were submitted to an accelerated fatigue-testing protocol [29,31] by a hydraulic testing machine (Instron ElektroPlus E3000, Norwood, MA,USA) at an angle of 135 degrees to the long axis of each tooth. Cyclic isometric loading was applied on the triangular ridge of the buccal cusp of the tooth using a round-shaped metallic tip (with a diameter of $5 \mathrm{~mm}$ ). To aid the proper positioning of the testing tip, the palatal cusp was slightly reduced. The cyclic load was applied at a frequency of $5 \mathrm{~Hz}$, starting with gradually increasing static loading till $100 \mathrm{~N}$ in $5 \mathrm{~s}$, followed by cyclic loading in $100 \mathrm{~N}$ steps, up to $1000 \mathrm{~N}, 5000$ cycles per step. The specimens were loaded until fracture occurred or 50,000 cycles were reached. The total number of survived cycles was recorded for each specimen for the survival analyses.

After recording failure load, each specimen was visually examined for the type and location of the failure, as well as the direction of failure. According to Scotti and co-workers, a distinction was made between restorable and non-restorable fractures under an optical microscope (Carl Zeiss Omni Pico, Oberkochen, Germany) with a two-examiner agreement. A restorable fracture is above the CEJ, meaning that in case of fracture, the tooth can be restored, while a nonrestorable fracture extends below the CEJ, and the tooth is likely to be extracted [36].

Statistical analysis was performed in SPSS 23.0 (IBM Corp., Somers, NY, USA). Kaplan-Meyer survival analysis was conducted, followed by pairwise post hoc comparisons (Breslow). The frequency of restorable and non-restorable fractures was calculated for each group. 




Fig. 2 Fatigue resistance survival curves (Kaplan-Meier survival estimator) for all tested groups

Table 2 Average of survived load cycles and their standard deviations

\begin{tabular}{lllc}
\hline Group & Mean & $N$ & Std. Deviation \\
\hline Group A1 (control) & $11,590.55$ & 11 & 4316.577 \\
Group A2 & $16,527.92$ & 12 & $11,556.415$ \\
Group A3 & $20,296.00$ & 11 & 7683.110 \\
Group B1 & $14,621.82$ & 11 & 4628.846 \\
Group B2 & $16,699.75$ & 12 & 6214.033 \\
Group B3 & $23,735.55$ & 11 & 7933.014 \\
Group C1 & $22,124.82$ & 11 & 6782.084 \\
Group C2 & $21,739.92$ & 12 & 6380.115 \\
Group C3 & $30,728.50$ & 12 & 4358.524 \\
Total & $19,848.63$ & 103 & 8644.093
\end{tabular}

\section{Results}

The Kaplan-Meier survival curves are presented in Fig. 2. Table 2 shows the descriptive characterization of the survival as the mean and median number of survived cycles for each tested group. During the mechanical fatigue testing, the embedding surrounding the teeth broke in 5 specimens, but the teeth did not; thus, those teeth were excluded from the study. Table 3 shows the $p$-values for the group-wise comparisons. Group C3 presented the highest survival rate, while the control group presented the lowest survival rate in this study. Furthermore, Group C3 showed significantly higher survival values compared to all other tested groups $(p<0.05)$. The control group did

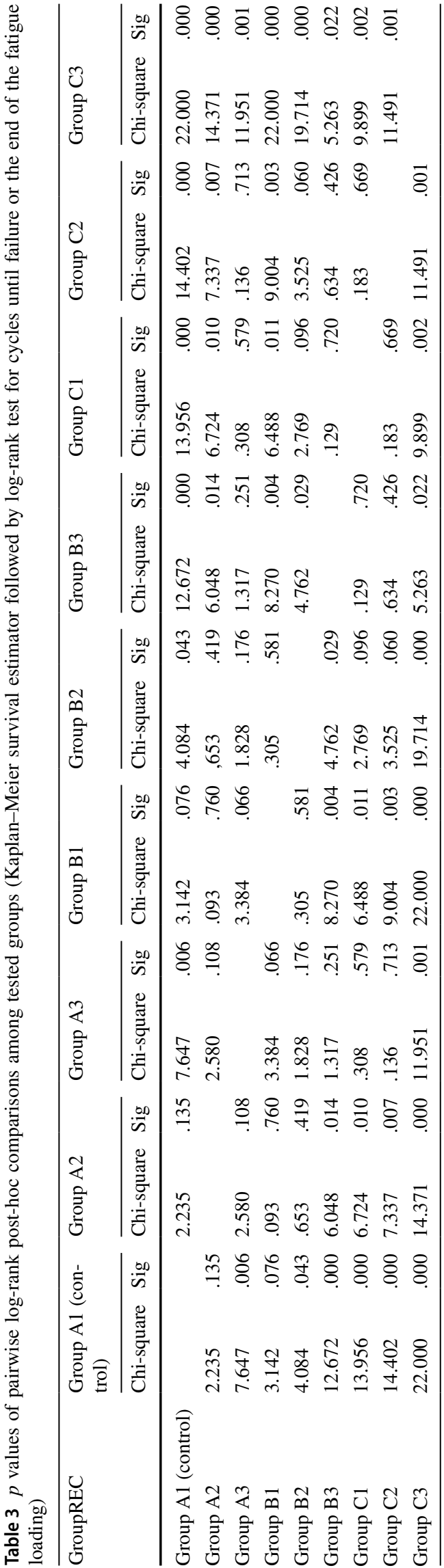


not differ statistically from Groups B1 and A2 $(p=0.076$ and $p=0.135$, respectively). The rest of the tested groups showed statistically significantly higher survival rates compared to the control group $(p<0.05)$. Group B3 showed higher survival rates than the control group, and Groups A2, B1, and B2 $(p<0.05)$, however, did not differ from Groups A3, C1, and C2 in terms of survival.

Regarding the type of fractures, all restored groups showed dominantly non-restorable fractures (Table 4, Fig. 3). Non-restorable fractures ended either (a) below or at the bone level emerging from the buccal or palatal wall or (b) spreading into the root splitting the tooth. Interrater agreement regarding fracture patterns was assessed by Cohen's $\kappa$. As there was an extremely low number of cases $(N=3)$ where the raters did not agree, and this meant that in most of the groups the agreement was $100 \%$, calculated kappa was calculated for the entire sample. The test indicated almost perfect agreement $(\kappa=0.93)$. The highest number of non-restorable fractures was observed in Group C3.

\section{Discussion}

This study focused on the effect of individualized fiber-reinforced designs with and without cuspal coverage on fatigue survival. The question of whether direct cuspal coverage can be as effective as its indirect counterpart was also addressed.

In our study, cyclic loading was used instead of static load-to-fracture testing. When testing tooth-restoration units, cycling loading, by generating repetitive forces just as during normal chewing, corresponds better to the real clinical situation. Also, as stated by Le Bell-Rönnlöf, fatigue more often leads to root fracture compared to static forces [27]. Accelerated fatigue testing has been used in many studies [37-43]. Accelerated fatigue was introduced as a rational middle ground between the load-to-fracture test and the more sophisticated and time-consuming fatigue tests [44]. In this study, maxillary RCT premolars with MOD cavities were used as they present unfavorable anatomy in crown volume and crown-to-root proportion, making them more

Table 4 The distribution of fracture mode among the tested groups

\begin{tabular}{lllllllllll}
\hline & & $\begin{array}{l}\text { Group A1 } \\
\text { (control) }\end{array}$ & Group A2 & Group A3 & Group B1 & Group B2 & Group B3 & Group C1 & Group C2 & Group C3 \\
\hline Non-restorable & Count & 9 & 8 & 10 & 9 & 9 & 6 & 8 & 7 & 11 \\
& \% within group & $81.8 \%$ & $66.7 \%$ & $90.9 \%$ & $81.8 \%$ & $75.0 \%$ & $54.5 \%$ & $72.7 \%$ & $58.3 \%$ & $91.7 \%$ \\
\multirow{2}{*}{ Restorable } & Count & 2 & 4 & 1 & 2 & 3 & 5 & 3 & 5 & 1 \\
& \% within group & $18.2 \%$ & $33.3 \%$ & $9.1 \%$ & $18.2 \%$ & $25.0 \%$ & $45.5 \%$ & $27.3 \%$ & $41.7 \%$ & $8.3 \%$ \\
\hline
\end{tabular}

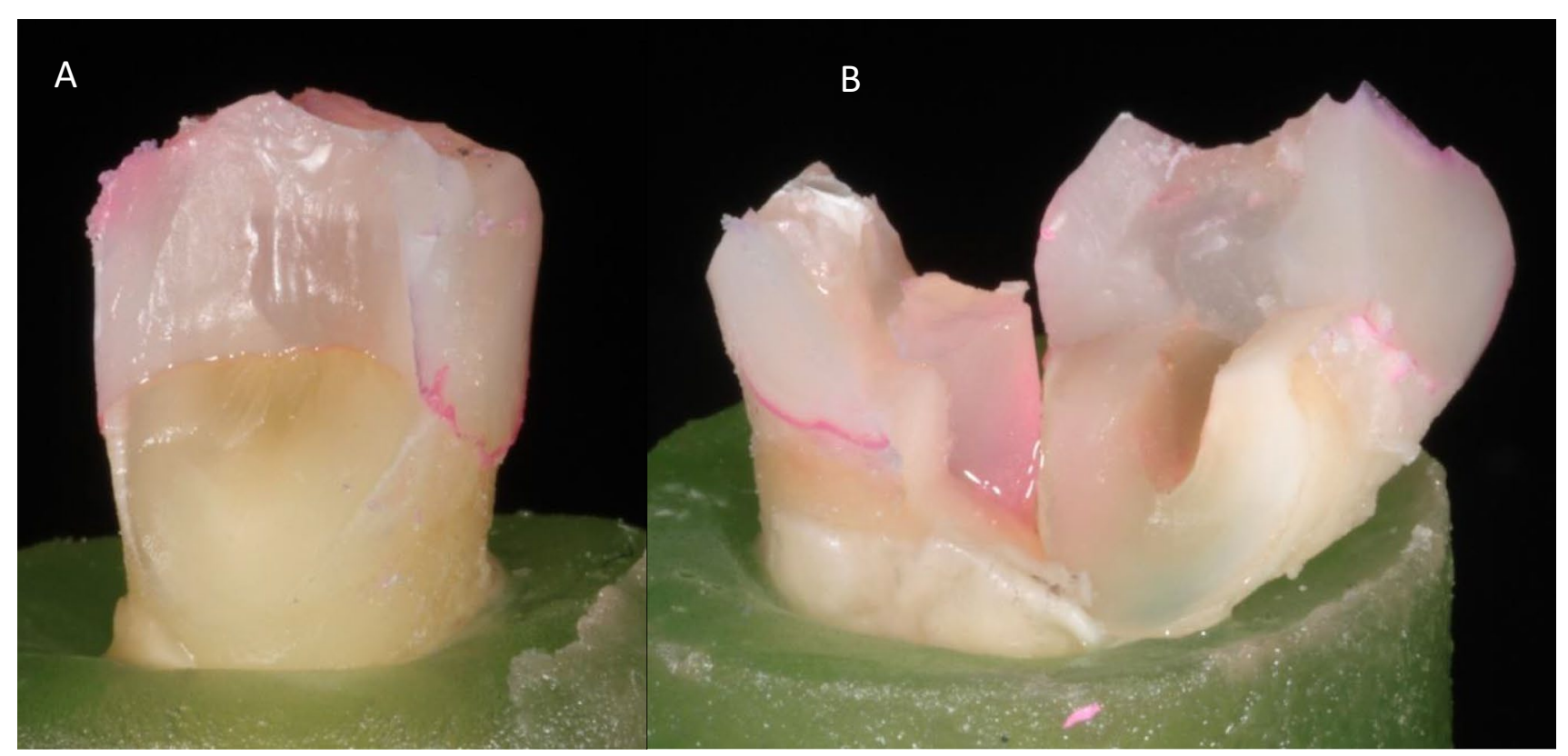

Fig. 3 Photographs of restorable (A) and non-restorable (B) fracture mode of the tested specimens 
susceptible to cusp fractures compared to other posterior teeth when exposed to occlusal load $[16,45]$. Furthermore, during mastication, premolars are subjected to a more detrimental combination of lateral forces, namely, a combination of shear and compressive forces, which increases the risk of future cusp fracture [46]. In our study, the tested specimens received oblique loading, which appears to be the worstcase scenario in terms of the loading direction in mechanical testing of RCT teeth as described by Wandscher et al. [47].

In the past years, the Bioblock technique utilizing packable or flowable SFRC showed remarkable results in restoring root canal-treated teeth in our studies [28, 29]. However, when short and long fibers are combined for individualized reinforcement, so far superior results can be seen $[31,48$, 49].

Choosing the best material for core build-up in root canaltreated teeth is a hot, yet unsolved topic. In a current systematic review and meta-analysis, Zarow et al. concluded that highly filled core composite materials could be a better option compared to classic composite materials [50].

In contrary to this, Stavridakis et al. recommend the usage of flowable composites as a core build-up material next to an FRC post; however, they made this recommendation in connection with anterior cases without a ferrule [51]. Fiberreinforced composites outperformed non-fiber-reinforced composites in many cavity formations and situations. Furthermore, since flowable SFRC has shown superior mechanical features compared to packable SFRC [52], it seems logical to use the flowable variant also as a core build-up either alone or next to an FRC post. In the study of Lassila et al. and Uctasli et al., flowable SFRC yielded promising results when used as a core build-up together with an FRC post $[48,53]$. More importantly from a clinical point of view, the handling and application of the flowable SFRC are much more convenient for a core build-up either alone or together with an FRC post compared to packable composites, be they fiber-reinforced or not.

In our study, the control group (composite filling) was characterized by the lowest and group C3 (indirect overlay placed on a core containing both short and long fibers) with the highest survival rates. When looking at the direct restorations without cuspal coverage in this study, Group A3 (flowable SFRC with an individually made FRC post) showed superior survival compared to the control group $(p=0.006)$. Thus, the first null hypothesis was rejected. This is in line with our previous findings [31] in RCT premolars with mesio-occlusal (MO) cavities. In this study, the Bioblock technique without cuspal coverage (Group A2) was not significantly superior to the non-fiber-reinforced composite filling (control group). This is apparently contrary to the findings of Shah et al. [54] and Eapen et al. [45]. However, they used SFRC only in the coronal cavity and performed load-to-fracture testing. Garlapati and colleagues also found
SFRC to yield significantly higher fracture resistance values in RCT MOD cavities compared to PFC composite filling [55]. However, they used a load-to-fracture test, and the tested specimens were molar teeth, which could explain the difference.

As for the optimal treatment of RCT MOD cavities, the literature seems to agree that indirect cuspal coverage is indicated for safety reasons [32-34]. However, with the advancement of adhesive techniques and composite materials, the possibility of direct cuspal coverage with PFC has arisen. This technique eliminates the need for a dental technician, making the restorative procedure less timeconsuming and less expensive. Direct cuspal coverage as a successful restorative option has been documented in clinical settings [56-58]. When considering the results on direct cuspal coverage, direct cuspal coverage on PFC composite filling (Group B1) did not prove to be significantly more beneficial than the methods without cuspal coverage (Control, Groups A2-3). This is in agreement with the results of Mohammadi et al. [2] but contradicts the findings of Fennis et al. [59] and ElAyouti et al. [60]. Interestingly, when direct cuspal coverage was combined with the Bioblock technique (Group B2), the survival significantly improved as compared to the control group $(p=0.043)$. It seems obvious that this can be put down to the strengthening effect of incorporating flowable SFRC into the restoration and providing cuspal coverage simultaneously. It is noteworthy that not only did the Bioblock technique with direct cuspal coverage (Group B2) not differ from the custom-made FRC post with flowable SFRC (Group A3) in terms of survival, but it was not significantly different from the indirect cuspal coverage restorations on either composite (Group C1) or Bioblock core build-up (Group C2) either. These results could suggest that the Bioblock technique combined with direct cuspal coverage or a custom-made FRC post with flowable SFRC without cuspal coverage could be alternatives to indirect cuspal coverage. To our knowledge, this is a novel finding under in vitro conditions. These FRC-based approaches can provide a safe direct restorative solution for patients with RCT MOD cavities in the premolar area. While there was no statistically significant difference between the Bioblock technique and the custom-made FRC post with flowable SFRC when not using cuspal coverage (Groups A2 and A3), a significant difference was found when direct cuspal coverage was applied with the same two FRC-based approaches (Groups B2 and B3, $p=0.029$ ). It seems that the use of long fibers in the form of a custom-made FRC post (everSTICK POST) is beneficial in premolar RCT teeth. This is in line with our previous findings [28, 31]. The explanation is probably that long fibers provide additional protection against the compressive, shear, and even tensile forces that premolars must withstand due to their position in the arch. Thus, it seems that in regions where teeth are potentially exposed to 
high shear and tensile forces (typically the anterior and premolar regions), it is recommendable to include long fibers in the restoration of RCT teeth [49]. However, these recommendations should be verified under in vivo conditions in the future.

It is especially important to note that while the restored teeth are in function, damage-causing tensile stress occurs especially on their outer surface, so the tougher material(s) should be placed there rather than in the middle of the root canal where the neutral axis of stress is located. This approach, i.e., the individual fiber post concept, has been used successfully with continuous long glass fibers [61]. Short fiber-reinforced core composites, on the other hand, showed the ability to re-direct and stop crack propagation within the materials [31]. The presence of such energyabsorbing and stress-distributing fibers allows crack propagation to be deflected away from the bulk of the material and toward the peripheries [62-64].

In this study, individualized fiber-reinforced post and cores were placed 6-mm deep into the root canal, as proposed in our most recent study [31]. So far many studies have pointed out that the length of FRC posts inside the canal is not a critical factor [65-68], in contrast to the situation when metal posts are used. According to Meyenberg, it could be enough for a clinically satisfactory outcome to have approximately $7 \mathrm{~mm}$ of FRC post inserted inside the canal with approximately $4 \mathrm{~mm}$ of the same post providing the coronal retention to the core build-up [69].

This could even be more relevant in the case of individualized FRC post and cores as they fit better in the critical cervical area in the root canal (since the amount of fibers are maximized while the luting cement is minimized). Subsequently, the increased amount of fibers can potentially compensate for the reduced post insertion depth in these cases. It is to be pointed out that the 6-mm post depth in this study was chosen on purpose, to allow direct comparison with the results of our previous study on restoring ET premolar teeth. The authors consider this a strength of this study.

Although there is evidence on the concept of using shorter (up to $7 \mathrm{~mm}$ ) individualized in the cervical part FRC posts, it has to be very clear that we are talking about single tooth restoration receiving inlays/onlays or crowns. The efficacy of this type of post and core to be used in endodontically treated abutment teeth of fixed partial dentures, especially the long span or in abutments of removable partial dentures, is not supported by the current data and warrants further investigation.

Direct cuspal coverage did not improve the fatigue survival of specimens restored with the custom-made FRC post with flowable SFRC (Group B3 compared to A3). This could be attributed to the unique properties that emerge as the custom-made RFC post and the flowable SFRC are combined. The custom-made FRC post is made of unidirectional fibers (E-glass) impregnated with a combination of bisphenol A-glycidyl methacrylate as the cross-linked phase and polymethyl methacrylate as a linear phase, forming together a semi-interpenetrating polymer network (semi-IPN) [70]. Since this semi-IPN structure contains both cross-linked and linear polymer phases, it ensures a stable adhesion to resin materials used for luting. In Groups A3, B3, and C3, flowable SFRC was used for post luting. This is an important aspect as this way either long (FRC post) or short fibers (flowable SFRC) will be in direct contact with the wall of the root canal, where the detrimental stress occurs upon loading $[27,71]$. According to our previous studies, SFRC materials inside the root canal, including flowable SFRC used for luting next to an individual FRC post, can be safely lightcured $[28,29,31]$. This could be traced back to multiple reasons, namely, the light transmission of the FRC post [72], the transparency of the SFRC materials, and the scattering of light by the short fibers [73]. As a result, SFRC materials can be safely light-cured to a depth of approximately $5 \mathrm{~mm}$ even in cavities and without the aid of an FRC post [74-76].

The highest survival rate was seen when indirect cuspal coverage was provided with the individual FRC post and flowable SFRC combination (Group C3). More importantly, this restorative method led to significantly better survival rates compared to all the other approaches. Within our study setup, this was the only case when a significant difference was found between direct and indirect cuspal coverage restorations utilizing fiber-reinforced core build-ups.

Regarding the fracture patterns, all groups were characterized by predominantly non-restorable fractures. Therefore, the second null hypothesis was accepted. It is clearly shown in the literature that the brittleness of the conventional PFC materials generates bulk fracture propagating easily through the whole thickness of the restoration, reaching the tooth substance to be propagated further $[62,63]$. Thus, the basic characteristics of the PFC materials do not offer significant protection against fatigue crack propagation. In contrast, the SFRC core supports the PFC layer and acts as a crack-stopper $[35,62,63]$. To be able to reinforce PFC, the integral toughness of the SFRC-core should be superior to that of the PFC surface layer [77]. If the SFRC-core is considered primarily a crack-stopper, it is the distance between the stress initiation point on the surface and the SFRC-core that matters. Thus, the top surface PFC thickness might contribute to the fatigue survival and failure mode. In this study, a 2-mm thick PFC surface layer was utilized, and this might explain the non-restorable fracture pattern even in the SFRC-core groups. This is in line with the results of previous investigations which showed the importance of SFRC and PFC layer thickness [63, 77, 78].

To maximize the amount of fibers and toughness, flowable SFRC (dentine shade) was used to build up the missing interproximal walls in Groups A2, A3, B2, and B3. It is to 
be noted that SFRC is supposed to be used as a bulk base or core foundation and should not be used as final surface restoration, as per the manufacturer's instructions. At the same time, several laboratory investigations and clinical reports showed that the filler loading of these materials did not affect either the wear or the gloss of the composite restorations [52, 79-82].

This was an in vitro study carried out on extracted human teeth. While the study has several strengths, it also has its limitations. For instance, the dynamic loading tests were not carried out in a fluid chamber, which would have modeled the intraoral environment more closely. The authors are aware of this limitation, and the reader is advised to interpret the results with this in mind. Furthermore, our results need to be verified in vivo, and other aspects should also be examined, such as wear, color stability, and the quality of interproximal contacts in time. Finally, ceramic overlays should also be tested on the proposed individualized fiberreinforced post and core designs.

\section{Conclusions}

Custom-made fiber post and SFRC as post luting core material with or without cuspal coverage performed well in terms of fatigue resistance and survival when used for the restoration of ET premolars.

The performance of indirect CAD/CAM overlay restorations was superior to direct composite overlay restorations.

Acknowledgements The authors would like to thank Roland Temple dental technician for his valuable work in fabricating the indirect overlays.

Funding Open Access funding provided by University of Turku (UTU) including Turku University Central Hospital. This study was supported by the EFOP 3.6.3-VEKOP-16-2017-00009, by the Bolyai János Research Scholarship (BO/701/20/5), and by the ÚNKP-20-3-SZTE, ÚNKP-20-5-SZTE New National Excellence Program of the Ministry for Innovation and Technology from the Source of National Research, Development and Innovation Fund, Hungary.

\section{Declarations}

Ethical approval This article does not contain any studies with human participants or animals performed by any of the authors.

Conflict of interest Author Márk Fráter declares that he has no conflict of interest. Author Tekla Sáry declares that he has no conflict of interest. Author Janka Molnár declares that he has no conflict of interest. Author Gábor Braunitzer declares that he has no conflict of interest. Author Lippo Lassila declares that he has no conflict of interest. Author Pekka Vallittu declares that he consults Stick Tech - Member of GC in training and RD. Author Sufyan Garoushi declares that he has no conflict of interest.
Informed consent For this type of study, formal consent is not required.

Open Access This article is licensed under a Creative Commons Attribution 4.0 International License, which permits use, sharing, adaptation, distribution and reproduction in any medium or format, as long as you give appropriate credit to the original author(s) and the source, provide a link to the Creative Commons licence, and indicate if changes were made. The images or other third party material in this article are included in the article's Creative Commons licence, unless indicated otherwise in a credit line to the material. If material is not included in the article's Creative Commons licence and your intended use is not permitted by statutory regulation or exceeds the permitted use, you will need to obtain permission directly from the copyright holder. To view a copy of this licence, visit http://creativecommons.org/licenses/by/4.0/.

\section{References}

1. Al-Omiri MK, Mahmoud AA, Rayyan MR, Abu-Hammad O (2010) Fracture resistance of teeth restored with post-retained restorations: an overview. J Endod 36(9):1439-1449. https:// doi.org/10.1016/j.joen.2010.06.005

2. Mohammadi N, Kahnamoii MA, Yeganeh PK, Navimipour EJ (2009) Effect of fiber post and cusp coverage on fracture resistance of endodontically treated maxillary premolars directly restored with composite resin. J Endod 35(10):1428-1432. https://doi.org/10.1016/j.joen.2009.07.010

3. Barcellos RR, Correia DP, Farina AP, Mesquita MF, Ferraz CC, Cecchin D (2013) Fracture resistance of endodontically treated teeth restored with intra-radicular post: the effects of post system and dentine thickness. J Biomech 46(15):25722577. https://doi.org/10.1016/j.jbiomech.2013.08.016

4. Dietschi D, Duc O, Krejci I, Sadan A (2007) Biomechanical considerations for the restoration of endodontically treated teeth: a systematic review of the literature-Part 1. Composition and micro- and macrostructure alterations. Quintessence Int 38(9):733-743

5. Taha NA, Palamara JE, Messer HH (2011) Fracture strength and fracture patterns of root filled teeth restored with direct resin restorations. J Dent 39(8):527-535. https://doi.org/10.1016/j. jdent.2011.05.003

6. Rocca GT, Krejci I (2013) Crown and post-free adhesive restorations for endodontically treated posterior teeth: from direct composite to endocrowns. Eur J Esthet Dent 8(2):156-179

7. Tay FR, Pashley DH (2007) Monoblocks in root canals: a hypothetical or a tangible goal. J Endod 33(4):391-398. https://doi. org/10.1016/j.joen.2006.10.009

8. El-Helali R, Dowling AH, McGinley EL, Duncan HF, Fleming GJ (2013) Influence of resin-based composite restoration technique and endodontic access on cuspal deflection and cervical microleakage scores. J Dent 41(3):216-222. https://doi.org/10. 1016/j.jdent.2012.11.002

9. Reeh ES, Douglas WH, Messer HH (1989) Stiffness of endodontically-treated teeth related to restoration technique. J Dent Res 68(11):1540-1544. https://doi.org/10.1177/002203458906801 11401

10. Wu Y, Cathro P, Marino V (2010) Fracture resistance and pattern of the upper premolars with obturated canals and restored endodontic occlusal access cavities. J Biomed Res 24(6):474478. https://doi.org/10.1016/S1674-8301(10)60063-2

11. Hannig C, Westphal C, Becker K, Attin T (2005) Fracture resistance of endodontically treated maxillary premolars restored with CAD/CAM ceramic inlays. J Prosthet Dent 94(4):342-349. https://doi.org/10.1016/j.prosdent.2005.08.004 
12. Seo DG, Yi YA, Shin SJ, Park JW (2012) Analysis of factors associated with cracked teeth. J Endod 38(3):288-292. https:// doi.org/10.1016/j.joen.2011.11.017

13. Oskoee PA, Ajami AA, Navimipour EJ, Oskoee SS, Sadjadi J (2009) The effect of three composite fiber insertion techniques on fracture resistance of root-filled teeth. J Endod 35(3):413416. https://doi.org/10.1016/j.joen.2008.11.027

14. Belli S, Erdemir A, Yildirim C (2006) Reinforcement effect of polyethylene fibre in root-filled teeth: comparison of two restoration techniques. Int Endod J 39(2):136-142. https://doi. org/10.1111/j.1365-2591.2006.01057.x

15. Zicari F, De Munck J, Scotti R, Naert I, Van Meerbeek B (2012) Factors affecting the cement-post interface. Dent Mater 28(3):287-297. https://doi.org/10.1016/j.dental.2011.11.003

16 Soares PV, Santos-Filho PC, Martins LR, Soares CJ (2008) Influence of restorative technique on the biomechanical behavior of endodontically treated maxillary premolars. Part I: fracture resistance and fracture mode. J Prosthet Dent 99(1):30-37. https://doi.org/10.1016/S0022-3913(08)60006-2

17. Scotti N, Scansetti M, Rota R, Pera F, Pasqualini D, Berutti E (2011) The effect of the post length and cusp coverage on the cycling and static load of endodontically treated maxillary premolars. Clin Oral Investig 15(6):923-929. https://doi.org/10.1007/ s00784-010-0466-y

18. Nothdurft FP, Seidel E, Gebhart F, Naumann M, Motter PJ, Pospiech PR (2008) The fracture behavior of premolar teeth with class II cavities restored by both direct composite restorations and endodontic post systems. J Dent 36(6):444-449. https://doi.org/ 10.1016/j.jdent.2008.03.004

19. Trope M, Maltz DO, Tronstad L (1985) Resistance to fracture of restored endodontically treated teeth. Endod Dent Traumatol 1(3):108-111. https://doi.org/10.1111/j.1600-9657.1985.tb005 71.x

20. Zicari F, Van Meerbeek B, Scotti R, Naert I (2012) Effect of fibre post length and adhesive strategy on fracture resistance of endodontically treated teeth after fatigue loading. J Dent 40(4):312321. https://doi.org/10.1016/j.jdent.2012.01.006

21. Soares CJ, Soares PV, de Freitas Santos-Filho PC, Castro CG, Magalhaes D, Versluis A (2008) The influence of cavity design and glass fiber posts on biomechanical behavior of endodontically treated premolars. J Endod 34(8):1015-1019. https://doi.org/10. 1016/j.joen.2008.05.017

22. Stockton L, Lavelle CL, Suzuki M (1998) Are posts mandatory for the restoration of endodontically treated teeth? Endod Dent Traumatol 14(2):59-63. https://doi.org/10.1111/j.1600-9657. 1998.tb00810.x

23. Faria-e-Silva AL, Pedrosa-Filho Cde F, Menezes Mde S, Silveira DM, Martins LR (2009) Effect of relining on fiber post retention to root canal. J Appl Oral Sci 17(6):600-604. https://doi.org/10. 1590/s1678-77572009000600012

24. D’Arcangelo C, Cinelli M, De Angelis F, D'Amario M (2007) The effect of resin cement film thickness on the pullout strength of a fiber-reinforced post system. J Prosthet Dent 98(3):193-198. https://doi.org/10.1016/S0022-3913(07)60055-9

25. Bell AM, Lassila LV, Kangasniemi I, Vallittu PK (2005) Bonding of fibre-reinforced composite post to root canal dentin. J Dent 33(7):533-539. https://doi.org/10.1016/j.jdent.2004.11.014

26. Chieruzzi M, Pagano S, Pennacchi M, Lombardo G, D'Errico P, Kenny JM (2012) Compressive and flexural behaviour of fibre reinforced endodontic posts. J Dent 40(11):968-978. https://doi. org/10.1016/j.jdent.2012.08.003

27. Le Bell-Rönnlöf AM, Lassila LV, Kangasniemi I, Vallittu PK (2011) Load-bearing capacity of human incisor restored with various fiber-reinforced composite posts. Dent Mater 27(6):107-115. https://doi.org/10.1016/j.dental.2011.02.009
28. Fráter M, Lassila L, Braunitzer G, Vallittu PK, Garoushi S (2020) Fracture resistance and marginal gap formation of post-core restorations: influence of different fiber-reinforced composites. Clin Oral Investig 24(1):265-276. https://doi.org/10.1007/s00784-01902902-3 (Erratum in: Clin Oral Investig 25(5):3339-3340)

29. Fráter M, Sáry T, Néma V, Braunitzer G, Vallittu P, Lassila L, Garoushi S (2021) Fatigue failure load of immature anterior teeth: influence of different fiber post-core systems. Odontology 109(1):222-230. https://doi.org/10.1007/s10266-020-00522-y

30. Fráter M, Sáry T, Garoushi S (2020) Bioblock technique to treat severe internal resorption with subsequent periapical pathology: a case report. Restor Dent Endod 45(4):e43. https://doi.org/10. 5395/rde.2020.45.e43

31. Fráter M, Sáry T, Jókai B, Braunitzer G, Säilynoja E, Vallittu PK, Lassila L, Garoushi S (2021) Fatigue behavior of endodontically treated premolars restored with different fiber-reinforced designs. Dent Mater 37(3):391-402. https://doi.org/10.1016/j.dental.2020. 11.026

32. Sorensen JA, Martinoff JT (1984) Intracoronal reinforcement and coronal coverage: a study of endodontically treated teeth. J Prosthet Dent 51(6):780-784. https://doi.org/10.1016/0022-3913(84) 90376-7

33. Scotti N, Eruli C, Comba A, Paolino DS, Alovisi M, Pasqualini D, Berutti E (2015) Longevity of class 2 direct restorations in rootfilled teeth: a retrospective clinical study. J Dent 43(5):499-505. https://doi.org/10.1016/j.jdent.2015.02.006

34. Xie KX, Wang XY, Gao XJ, Yuan CY, Li JX, Chu CH (2012) Fracture resistance of root filled premolar teeth restored with direct composite resin with or without cusp coverage. Int Endod J 45(6):524-529. https://doi.org/10.1111/j.1365-2591.2011.02005.x

35. Fráter M, Forster A, Keresztúri M, Braunitzer G, Nagy K (2014) In vitro fracture resistance of molar teeth restored with a short fibre-reinforced composite material. J Dent 42(9):1143-1150. https://doi.org/10.1016/j.jdent.2014.05.004

36. Scotti N, Coero Borga FA, Alovisi M, Rota R, Pasqualini D, Berutti E (2012) Is fracture resistance of endodontically treated mandibular molars restored with indirect onlay composite restorations influenced by fibre post insertion? J Dent 40(10):814-820. https://doi.org/10.1016/j.jdent.2012.06.005

37. Lazari PC, de Carvalho MA, Del Bel Cury AA, Magne P (2018) Survival of extensively damaged endodontically treated incisors restored with different types of posts-and-core foundation restoration material. J Prosthet Dent 119(5):769-776. https://doi.org/10. 1016/j.prosdent.2017.05.012

38 Soares LM, Razaghy M, Magne P (2018) Optimization of large MOD restorations: composite resin inlays vs. short fiber-reinforced direct restorations. Dent Mater 34(4):587-597. https://doi. org/10.1016/j.dental.2018.01.004

39. Magne P, Lazari PC, Carvalho MA, Johnson T, Del Bel Cury AA (2017) Ferrule-effect dominates over use of a fiber post when restoring endodontically treated incisors: an in vitro study. Oper Dent 42(4):396-406. https://doi.org/10.2341/16-243-L

40. Gresnigt MMM, Özcan M, Carvalho M, Lazari P, Cune MS, Razavi P, Magne P (2017) Effect of luting agent on the load to failure and accelerated-fatigue resistance of lithium disilicate laminate veneers. Dent Mater 33(12):1392-1401. https://doi.org/ 10.1016/j.dental.2017.09.010

41. Goldberg J, Güth JF, Magne P (2016) Accelerated fatigue resistance of thick CAD/CAM composite resin overlays bonded with light- and dual-polymerizing luting resins. J Adhes Dent 18(4):341-348. https://doi.org/10.3290/j.jad.a36515

42. Magne P, Silva S, Andrada Md, Maia H (2016) Fatigue resistance and crack propensity of novel "super-closed" sandwich composite resin restorations in large MOD defects. Int J Esthet Dent 11(1):82-97 
43. Magne P, Schlichting LH, Maia HP, Baratieri LN (2010) In vitro fatigue resistance of CAD/CAM composite resin and ceramic posterior occlusal veneers. J Prosthet Dent 104(3):149-157. https:// doi.org/10.1016/S0022-3913(10)60111-4

44. Magne P, Carvalho AO, Bruzi G, Anderson RE, Maia HP, Giannini M (2014) Influence of no-ferrule and no-post buildup design on the fatigue resistance of endodontically treated molars restored with resin nanoceramic CAD/CAM crowns. Oper Dent 39(6):595-602. https://doi.org/10.2341/13-004-L

45. Eapen AM, Amirtharaj LV, Sanjeev K, Mahalaxmi S (2017) Fracture resistance of endodontically treated teeth restored with 2 different fiber-reinforced composite and 2 conventional composite resin core buildup materials: an in vitro study. J Endod 43(9):1499-1504. https://doi.org/10.1016/j.joen.2017.03.031

46. Robbins JW (2002) Restoration of the endodontically treated tooth. Dent Clin North Am 46(2):367-384. https://doi.org/10. 1016/s0011-8532(01)00006-4

47. Wandscher VF, Bergoli CD, Limberger IF, Ardenghi TM, Valandro LF (2014) Preliminary results of the survival and fracture load of roots restored with intracanal posts: weakened vs nonweakened roots. Oper Dent 39(5):541-555. https://doi.org/10. 2341/12-465

48. Lassila L, Oksanen V, Fráter M, Vallittu PK, Garoushi S (2020) The influence of resin composite with high fiber aspect ratio on fracture resistance of severely damaged bovine incisors. Dent Mater J 39(3):381-388. https://doi.org/10.4012/dmj.2019-051

49. Fráter M, Sáry T, Braunitzer G, Balázs Szabó P, Lassila L, Vallittu PK, Garoushi S (2021) Fatigue failure of anterior teeth without ferrule restored with individualized fiber-reinforced post-core foundations. J Mech Behav Biomed Mater 118:104440. https:// doi.org/10.1016/j.jmbbm.2021.104440

50. Zarow M, Dominiak M, Szczeklik K, Hardan L, Bourgi R, Cuevas-Suárez CE, Zamarripa-Calderón JE, Kharouf N, Filtchev D (2021) Effect of composite core materials on fracture resistance of endodontically treated teeth: a systematic review and metaanalysis of in vitro studies. Polymers (Basel) 13(14):2251. https:// doi.org/10.3390/polym13142251

51. Stavridakis M, Brokos Y, Krejci I (2018) Is the glass half empty or half full? A novel "philosophical" approach to the "mystery" of the so-called ferrule effect. Med Hypotheses 115:35-41. https:// doi.org/10.1016/j.mehy.2018.03.013

52. Lassila L, Keulemans F, Vallittu PK, Garoushi S (2020) Characterization of restorative short-fiber reinforced dental composites. Dent Mater J 39(6):992-999. https://doi.org/10.4012/dmj. 2019-088

53. Uctasli S, Boz Y, Sungur S, Vallittu PK, Garoushi S, Lassila L (2021) Influence of post-core and crown type on the fracture resistance of incisors submitted to quasistatic loading. Polymers (Basel) 13(7):1130. https://doi.org/10.3390/polym13071130

54. Shah S, Shilpa-Jain DP, Velmurugan N, Sooriaprakas C, Krithikadatta J (2020) Performance of fibre reinforced composite as a postendodontic restoration on different endodontic cavity designs- an in-vitro study. J Mech Behav Biomed Mater 104:103650. https:// doi.org/10.1016/j.jmbbm.2020.103650

55. Garlapati TG, Krithikadatta J, Natanasabapathy V (2017) Fracture resistance of endodontically treated teeth restored with short fiber composite used as a core material-an in vitro study. J Prosthodont Res 61(4):464-470. https://doi.org/10.1016/j.jpor.2017.02.001

56. Deliperi S, Bardwell DN (2006) Direct cuspal-coverage posterior resin composite restorations: a case report. Oper Dent 31(1):143150. https://doi.org/10.2341/04-177

57 Deliperi S, Bardwell DN (2008) Multiple cuspal-coverage direct composite restorations: functional and esthetic guidelines. J Esthet Restor Dent 20(5):300-8. https://doi.org/10.1111/j.1708-8240. 2008.00198.x (discussion 309-312)
58. Fennis WM, Kuijs RH, Roeters FJ, Creugers NH, Kreulen CM (2014) Randomized control trial of composite cuspal restorations: five-year results. J Dent Res 93(1):36-41. https://doi.org/10.1177/ 0022034513510946

59. Fennis WM, Kuijs RH, Kreulen CM, Verdonschot N, Creugers NH (2004) Fatigue resistance of teeth restored with cuspal-coverage composite restorations. Int J Prosthodont 17(3):313-317

60. ElAyouti A, Serry MI, Geis-Gerstorfer J, Löst C (2011) Influence of cusp coverage on the fracture resistance of premolars with endodontic access cavities. Int Endod J 44(6):543-549. https://doi. org/10.1111/j.1365-2591.2011.01859.x

61. Garoushi S, Tanner J, Keulemans F, Le Bell-Rönnlöf AM, Lassila L, Vallittu PK (2020) Fiber reinforcement of endodontically treated teeth: what options do we have? Literature review. Eur J Prosthodont Restor Dent 28(2):54-63. https://doi.org/10.1922/ EJPRD_2002Garoushi10

62. Lassila L, Säilynoja E, Prinssi R, Vallittu PK, Garoushi S (2020) Fracture behavior of Bi-structure fiber-reinforced composite restorations. J Mech Behav Biomed Mater 101:103444. https://doi. org/10.1016/j.jmbbm.2019.103444

63 Garoushi S, Sungur S, Boz Y, Ozkan P, Vallittu PK, Uctasli S, Lassila L (2021) Influence of short-fiber composite base on fracture behavior of direct and indirect restorations. Clin Oral Investig. https://doi.org/10.1007/s00784-020-03768-6 (online ahead of print)

64. Tiu J, Belli R, Lohbauer U (2020) Rising R-curves in particulate/ fiber-reinforced resin composite layered systems. J Mech Behav Biomed Mater 103:103537. https://doi.org/10.1016/j.jmbbm. 2019.103537

65. Ramírez-Sebastià A, Bortolotto T, Cattani-Lorente M, Giner L, Roig M, Krejci I (2014) Adhesive restoration of anterior endodontically treated teeth: influence of post length on fracture strength. Clin Oral Investig 18(2):545-554. https://doi.org/10. 1007/s00784-013-0978-3

66. Chuang SF, Yaman P, Herrero A, Dennison JB, Chang CH (2010) Influence of post material and length on endodontically treated incisors: an in vitro and finite element study. J Prosthet Dent 104(6):379-388. https://doi.org/10.1016/S0022-3913(10)60171-0

67. Dejak B, Młotkowski A (2013) The influence of ferrule effect and length of cast and FRC posts on the stresses in anterior teeth. Dent Mater 29(9):e227-e237. https://doi.org/10.1016/j.dental.2013.06. 002

68. Santos-Filho PC, Veríssimo C, Soares PV, Saltarelo RC, Soares CJ, Marcondes Martins LR (2014) Influence of ferrule, post system, and length on biomechanical behavior of endodontically treated anterior teeth. J Endod 40(1):119-123. https://doi.org/10. 1016/j.joen.2013.09.034

69. Meyenberg K (2013) The ideal restoration of endodontically treated teeth - structural and esthetic considerations: a review of the literature and clinical guidelines for the restorative clinician. Eur J Esthet Dent 8(2):238-268

70. Amižić IP, Miletić I, Baraba A, Fan Y, Nathanson D (2018) In vitro retention of prefabricated and individually formed posts: a pilot study. J Prosthet Dent 120(4):553-557. https://doi.org/10. 1016/j.prosdent.2018.04.011

71. Vallittu PK (2016) Are we misusing fiber posts? Guest editorial. Dent Mater 32(2):125-126. https://doi.org/10.1016/j.dental.2015. 11.001

72. Bell-Rönnlöf AL, Jaatinen J, Lassila L, Närhi T, Vallittu P (2019) Transmission of light through fiber-reinforced composite posts. Dent Mater J 38(6):928-933. https://doi.org/10.4012/dmj. 2018-217

73. Li X, Pongprueksa P, Van Meerbeek B, De Munck J (2015) Curing profile of bulk-fill resin-based composites. J Dent 43(6):664672. https://doi.org/10.1016/j.jdent.2015.01.002 
74. Garoushi S, Gargoum A, Vallittu PK, Lassila L (2018) Short fiberreinforced composite restorations: a review of the current literature. J Investig Clin Dent 9(3):e12330. https://doi.org/10.1111/ jicd. 12330

75. Lempel E, Őri Z, Kincses D, Lovász BV, Kunsági-Máté S, Szalma J (2021) Degree of conversion and in vitro temperature rise of pulp chamber during polymerization of flowable and sculptable conventional, bulk-fill and short-fibre reinforced resin composites. Dent Mater 37(6):983-997. https://doi.org/10.1016/j.dental.2021. 02.013

76. Lempel E, Ôri Z, Szalma J, Lovász BV, Kiss A, Tóth Á, KunságiMáté S (2019) Effect of exposure time and pre-heating on the conversion degree of conventional, bulk-fill, fiber reinforced and polyacid-modified resin composites. Dent Mater 35(2):217-228. https://doi.org/10.1016/j.dental.2018.11.017

77. Lassila L, Säilynoja E, Prinssi R, Vallittu PK, Garoushi S (2020) Bilayered composite restoration: the effect of layer thickness on fracture behavior. Biomater Investig Dent 7(1):80-85. https://doi. org/10.1080/26415275.2020.1770094

78. Garoushi S, Lassila LV, Tezvergil A, Vallittu PK (2006) Load bearing capacity of fibre-reinforced and particulate filler composite resin combination. J Dent 34(3):179-184. https://doi.org/10. 1016/j.jdent.2005.05.010
79. Lassila L, Säilynoja E, Prinssi R, Vallittu PK, Garoushi S (2020) The effect of polishing protocol on surface gloss of different restorative resin composites. Biomater Investig Dent 7(1):1-8. https://doi.org/10.1080/26415275.2019.1708201

80. Lassila L, Säilynoja E, Prinssi R, Vallittu P, Garoushi S (2019) Characterization of a new fiber-reinforced flowable composite. Odontology 107(3):342-352. https://doi.org/10.1007/ s10266-018-0405-y

81. Hosaka K, Tichy A, Hasegawa Y, Motoyama Y, Kanazawa M, Tagami J, Nakajima M (2020) Replacing mandibular central incisors with a direct resin-bonded fixed dental prosthesis by using a bilayering composite resin injection technique with a digital workflow: a dental technique. J Prosthet Dent S0022-3913(20):303759. https://doi.org/10.1016/j.prosdent.2020.05.007 (online ahead of print)

82. van Dijken JW, Sunnegårdh-Grönberg K (2006) Fiber-reinforced packable resin composites in class II cavities. J Dent 34(10):763769. https://doi.org/10.1016/j.jdent.2006.02.003

Publisher's note Springer Nature remains neutral with regard to jurisdictional claims in published maps and institutional affiliations. 